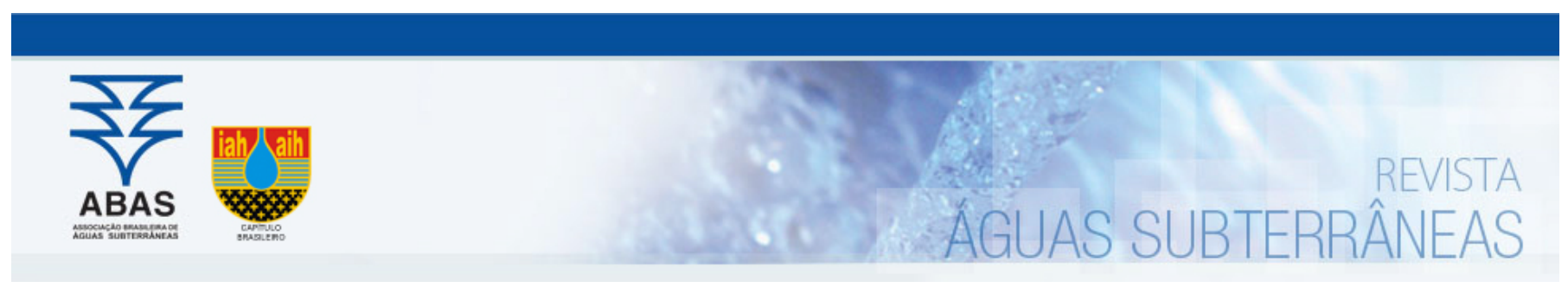

Artigos

\title{
Avaliação da vulnerabilidade natural à contaminação do sistema aquífero Serra Geral no Estado do Paraná - Brasil
}

\author{
Evaluation of natural vulnerability to contamination of Serra Geral aquifer \\ system In Paraná - Brazil
}

\author{
Vinícius Menezes Borges ${ }^{1}$; Gustavo Barbosa Athayde ${ }^{1}$; Pedro Antônio Roehe Reginato ${ }^{1}$ \\ 1 Universidade Federal do Rio Grande do Sul (UFRGS), Porto Alegre, RS, Brasil. \\ $\bowtie$ viniciusborges@ymail.com; gustavo.athayde@ufrgs.br; pedro.reginato@ufrgs.br
}

\begin{tabular}{|c|c|}
\hline & Resumo \\
\hline $\begin{array}{l}\text { Palavras-chave: } \\
\text { Vulnerabilidade. } \\
\text { DRASTIC. } \\
\text { GOD. } \\
\text { Sistema Aquífero Serra Geral. }\end{array}$ & $\begin{array}{l}\text { As águas subterrâneas são uma importante fonte de abastecimento humano, entretanto, estão se tornando cada vez mais } \\
\text { expostas à poluição devido à urbanização, desenvolvimento industrial e expansão agrícola. Assim, tornam-se necessárias me- } \\
\text { didas de proteção aos aquíferos. Neste trabalho é apresentado um estudo em escala regional sobre a vulnerabilidade natural } \\
\text { à contaminação das águas subterrâneas do Sistema Aquífero Serra Geral (SASG) no Estado do Paraná. A avaliação foi realizada } \\
\text { através dos métodos DRASTIC e GOD com adaptações para aplicação em estudos relacionados a aquíferos fraturados e os } \\
\text { métodos foram comparados através da normalização dos valores de vulnerabilidade.O mapa obtido através do método DRASTIC } \\
\text { apresentou graus de vulnerabilidade baixa }(5,01 \%) \text {, moderada }(83,13 \%) \text { e elevada }(11,85 \%) \text {. Já o método GOD apresentou } \\
\text { vulnerabilidades baixa ( } 66,21 \%) \text {, moderada }(0,67 \%) \text {, alta }(14,32 \%) \text { e extrema }(18,81 \%) \text {. O método DRASTIC apresentou maior } \\
\text { grau de detalhe na avaliação e mais valores intermediários. Já o método GOD apresentou variação brusca dos índices em muitas } \\
\text { regiões devido a parametrização simplificada. Apesar do método DRASTIC apresentar resultados mais satisfatórios, a escolha } \\
\text { do método deve estar condicionada à disponibilidade de dados e de recursos humanos e financeiros. }\end{array}$ \\
\hline
\end{tabular}

Abstract

Keywords:

Vulnerability.

DRASTIC.

GOD.

Serra Geral Aquifer System.

Groundwater is an important source for human supply, however, it is more and more exposed to contamination due to urbanization, industrial and agricultural expansion. This paper presents a study in regional scale about the natural vulnerability of groundwater in the Serra Geral Aquifer System in Paraná. DRASTIC and GOD methods with adaptations for fractured aquifers were used and compared through normalization of vulnerability values. The map obtained by DRASTIC method presented the following levels of vulnerability: low $(5,01 \%)$, moderate $(83,13 \%)$ and high $(11,85 \%)$. GOD method presented low $(66,21 \%)$, moderate $(0,67 \%)$, high $(14,32 \%)$ and extreme $(18,81)$ vulnerability. DRASTIC method presented better spatial detail and more intermediate values, while GOD showed abrupt due to the simple parameterization. Although the DRASTIC method presented better results, the choice for a method must be conditioned to the data, human and financial availability.

Revisado por pares.

Recebido em: 10/04/2017.

Aprovado em: 25/09/2017.

DOI: http://dx.doi.org/10.14295/ras.v31i4.28857

\section{INTRODUÇÃO}

As águas subterrâneas representam uma importante fonte de abastecimento e cerca de metade da população mundial depende exclusivamente deste recurso para o consumo (TUSHAAR et al., 2007). No Brasil, 37\% dos municípios são abastecidos por águas subterrâneas. No Estado do Paraná este percentual é ainda maior: $56 \%$ dos municípios são abastecidos exclusivamente por águas subterrâneas e 22\% por sistemas mistos.

Em decorrência da urbanização, do desenvolvimento industrial e expansão agrícola, os aquíferos estão cada vez mais expostos à contaminação, prejudicando a qualidade deste recurso. Assim, surge a necessidade de proteção das águas subterrâneas contra a poluição. Uma maneira para melhor orientar as medidas de proteção é realizar um mapeamento da vulnerabilidade natural das águas subterrâneas à contaminação, que está relacionada às características intrínsecas do aquífero, servindo como subsídio para a gestão do uso e ocupação do solo por parte da municipalidade, visando o planejamento e monitoramento em regiões mais vulneráveis.

Os primeiros mapeamentos da vulnerabilidade foram realizados por Albinet e Margat (1970) na escala 1:1.000.000 para todo território francês e mapas isolados nas escalas 1:250.000 e 1:50.000, com a finalidade de identificar áreas sensíveis à contaminação por ação humana. Segundo estes autores, as escalas dos mapas estão diretamente relacionadas com o grau de detalhe do estudo. Os mapas em escala menor (1:1.000.000) 
fornecem uma visão geral dos parâmetros hidrogeológicos que determinaram a vulnerabilidade em uma região, subsidiando as primeiras ações e medidas de precaução. Mapas em escala maior (1:250.000 e 1:50.000) fornecem uma visão mais detaIhada da área de estudo, podendo ser utilizados diretamente em estudos relacionados ao ordenamento do território e proteção da qualidade das águas subterrâneas.

Foster e Hirata (1988) definiram vulnerabilidade como a sensibilidade de um aquífero de ser negativamente afetado por uma carga contaminante aplicada na superfície em função de suas características intrínsecas. Essas características estão relacionadas à inacessibilidade hidráulica da zona saturada e a capacidade de atenuação dos estratos de cobertura, devido à retenção dos contaminantes no solo.

Para a determinação da vulnerabilidade das águas subterrâneas, diversos métodos são empregados, sendo alguns deles para contaminantes específicos e outros para contaminação em geral, tais como GOD, DRASTIC, SINTACS e IS (ALLER et al., 1987; FOSTER e HIRATA, 1988; CIVITA et al., 1994; FRANCÉS et al., 2001).A escolha do método está relacionada à disponibilidade de informações ou dados necessários para se aplicá-lo. Na existência de poucos dados, opta-se pela adoção de métodos mais simples e amplos, que podem ser utilizados de forma mais rápida e menos custosa (MENESES et al., 2009).

Neste contexto, este trabalho visa apresentar a avaliação da vulnerabilidade à contaminação do Sistema Aquífero Serra Geral (SASG) noParaná através dos métodos DRASTIC E GOD em escala regional, bem como comprar os dois métodos, investigando as semelhanças e diferenças apresentadas.

\section{CARACTERIZAÇÃO GEOLÓGICA E HIDROGEOLÓGICA DA ÁREA}

A área de estudo corresponde à porção aflorante da Formação Serra Geral no Paraná. Esta formação é representada por rochas vulcânicas resultantes de magmatismo mesozoico e recobre aproximadamente $75 \%$ da Bacia Sedimentar do Paraná. Quanto aos litotipos, predominam os basaltos com interposições de rochas ácidas (riolitos e riodacitos) (LICHT, 2013). No Brasil, sua área aflorante se estende do Rio Grande do Sul até o Sul de Minas Gerais (Figura 1).

O SASG possui grande importância para o estado do Paraná, uma vez que, segundo a SANEPAR (2015), 21\% da água tratada e consumida provém de aquíferos e o SASG contribui com 55\% deste volume. Abrange uma área de aproximadamente 109.000 $\mathrm{km}^{2}$ e sua espessura chega a $1347 \mathrm{~m}$ em Cianorte - PR, na região noroeste do estado. Possui contato com dois sistemas aquíferos: Aquífero Guarani (sotoposto) e Aquífero Caiuá (sobreposto). Quanto aos parâmetros hidráulicos, a profundidade do nível estático varia de 0 a 141,04 m, com valor médio de 18,08 m; capacidade específica média de 1,5 m³h-1m-1 e vazão média é de $21,5 \mathrm{~m}^{3} \mathrm{~h}-1$, sendo que, as maiores vazões são observadas em poços com profundidades entre 100 e $150 \mathrm{~m}$ (ATHAYDE e ATHAYDE, 2015).

O modelo conceitual utilizado na avaliação da vulnerabilidade considera que, regionalmente, o SASG se comporta como um aquífero livre, e sendo um aquífero fraturado, o fluxo da água está associado a estruturas tectônicas e atectônicas da Fm. Serra Geral. Recobrindo este aquífero, há um manto de alteração que possui espessuras diferentes, mas de grande importância para a recarga desse sistema. Assim, a água que precipita e infiltra pelas camadas de solo até atingir as estruturas presentes nas rochas, promovendo assim, a recarga desse sistema. Em função dos tipos de solos, suas propriedades físicas, bem como das condições de relevo e cobertura vegetal, haverá regiões onde a recarga vai ocorrer com maior ou menor intensidade. Soma-se o fato da presença do solo (geralmente argiloso) contribuir com a atenuação de eventuais contaminantes por processos de sorção, por exemplo.

\section{MATERIAL E MÉTODOS}

\subsection{Avaliação da vulnerabilidade pelo método DRASTIC}

O método DRASTIC (ALLER et al., 1987) para avaliação da vulnerabilidade fundamenta-se na soma ponderada de sete parâmetros: profundidade do nível estático (peso 5), recarga (peso 4), meio aquífero (peso 3), solo (peso 2), topografia (peso 1), impacto na zona vadosa (peso 5) e condutividade hidráulica (peso 3). Para cada parâmetro, existem faixas correspondentes a valores de um a dez, que multiplicados pelo respectivo peso dão origem a um Índice DRASTIC que pode variar entre $23 \mathrm{e}$ 230, indicando classes de vulnerabilidade baixa, intermediária, alta ou muito alta.

Para obtenção do índice de vulnerabilidade DRASTIC, cada um dos sete parâmetros do modelo foi multiplicado pelo seu respectivo peso mediante software de SIG ArcGIS 10.3. Em seguida, os valores foram reclassificados para que se enquadre nas faixas de vulnerabilidade definidas pelo método DRASTIC, que são: baixa (inferior a 120), moderada (entre 120 e 159), elevada (160 a 199) e muito elevada (maior que 199).

A profundidade do nível estático (D) foi obtida a partir de informações de 1625 poços tubulares perfurados no SASG (Figura 2). 0 mapa de nível estático foi gerado pela interpolação destes valores utilizando o método do inverso do quadrado da distância (IDW). A partir da reclassificação deste mapa, foi gerado o mapa do parâmetro $D$. 
Figura 1- Bacia do Paraná no Brasil e localização da área de estudo

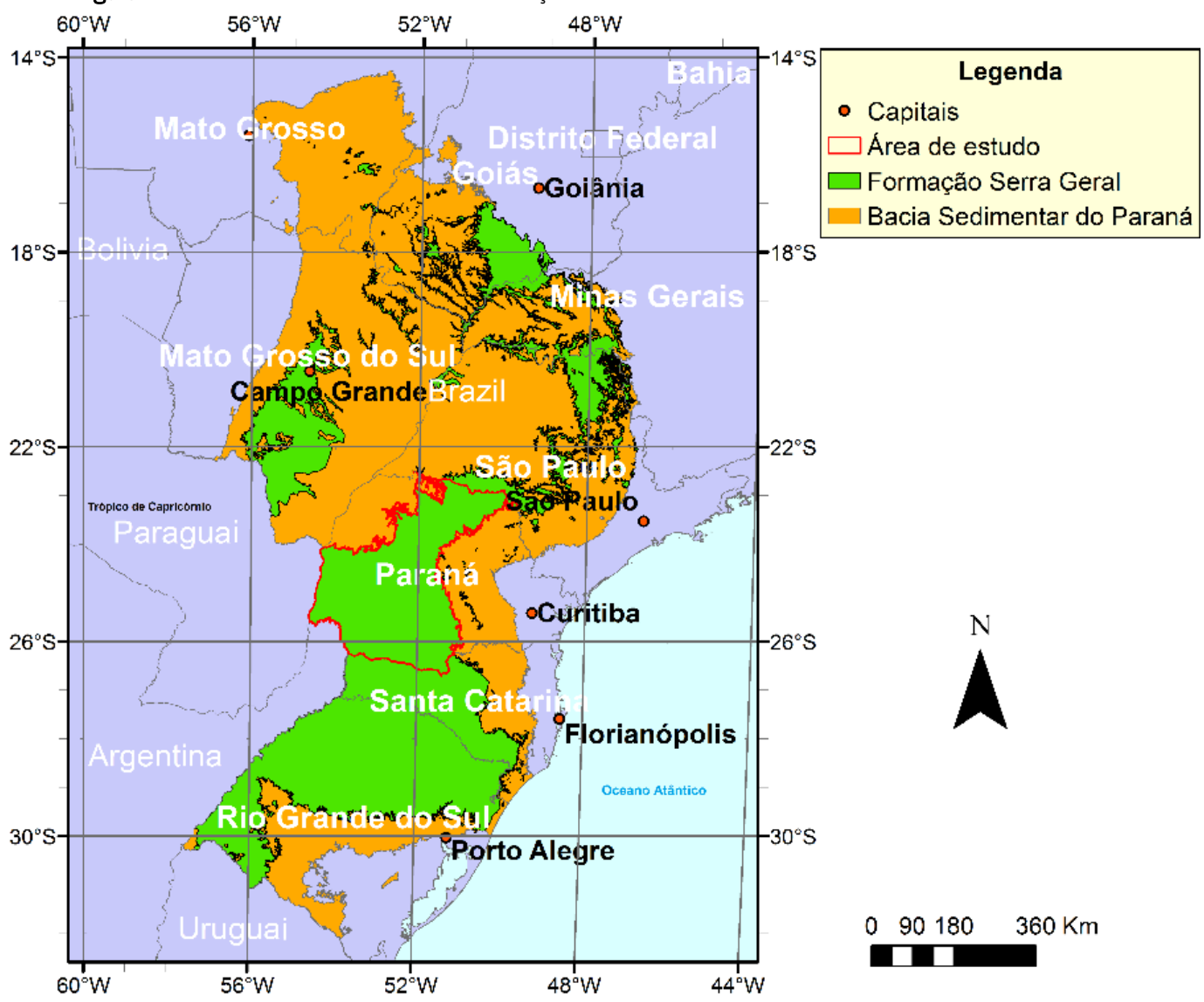

Figura 2 -Localização dos poços com valores de nível estático

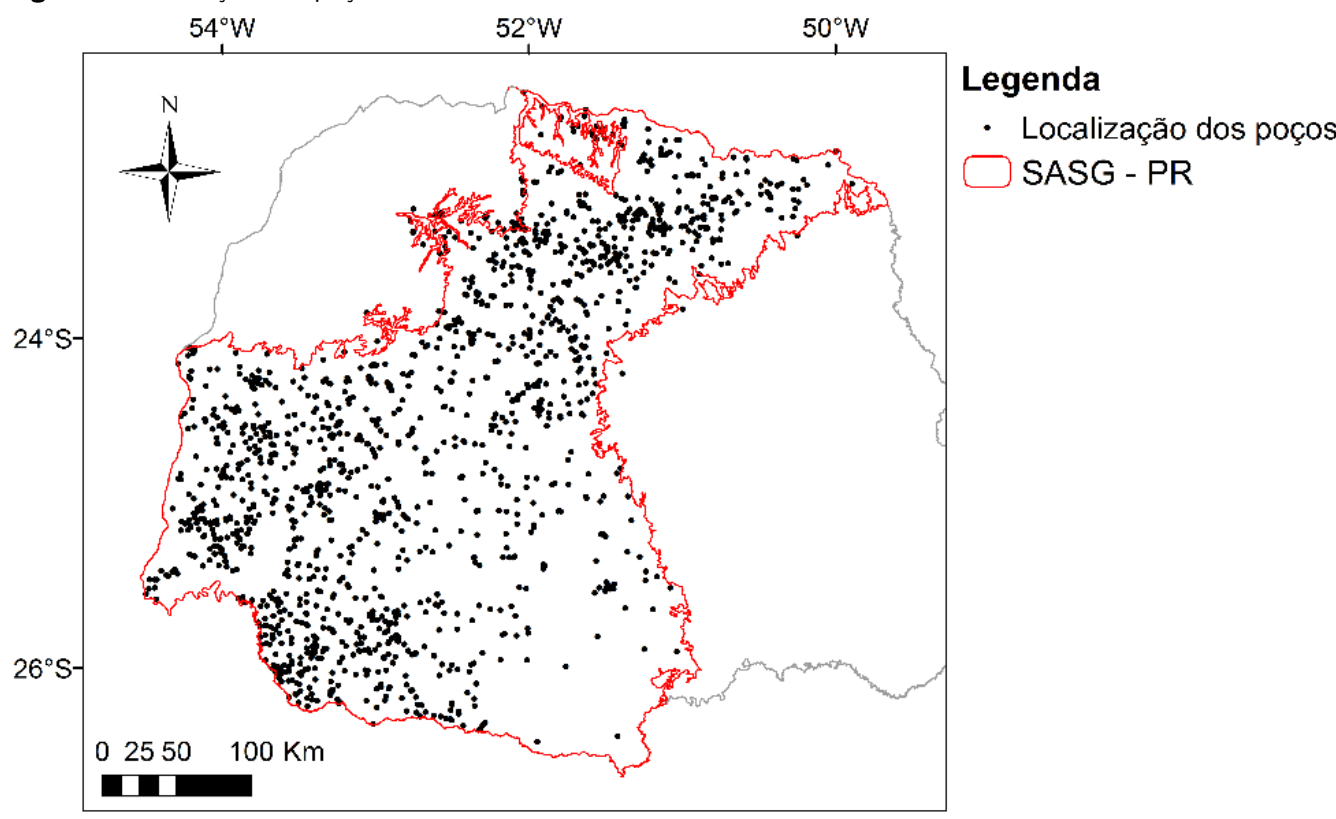

As taxas de recarga para cada sub-bacia que abrange o SASG foram calculadas por Borges (2017) por meio de balanço hídrico e da separação do escoamento de base utilizando o filtro digital de Eckhardt (2005). Estes são apresentados na Tabela 1. 
Tabela 1 - Taxas de recarga anual e índice DRASTIC por sub-bacias hidrográficas

\begin{tabular}{lccc}
\hline Sub-bacia & Área $\left(\mathrm{km}^{2}\right)$ & Recarga anual $(\mathrm{mm} / \mathrm{ano})$ & Índice DRASTIC \\
\hline Iguaçu & 54820,36 & 316 & 9 \\
Piquiri & 24171,67 & 489 & 9 \\
Ivaí & 36540,02 & 375 & 9 \\
Pirapó & 5098,12 & 284 & 9 \\
Tibagi & 24937,38 & 301 & 9 \\
Cinzas & 9612,78 & 175 & 6 \\
Paraná 3 & 7979,39 & 224 & 8 \\
Paranapanema 1 & 1231,65 & 170 & 6 \\
Paranapanema 2 & 663,83 & 123 & 6 \\
Paranapanema 3 & 3564,32 & 144 & 6 \\
\hline
\end{tabular}

A determinação do parâmetro A (meio aquífero) foi realizada mediante uma adaptação ao método. 0 modelo atribui índices de dois a dez para basalto, cuja variação depende do grau de faturamento da rocha, uma vez que este favorece a migração de contaminantes. Uma aproximação foi realizada partindo da densidade de lineamentos, em que, quanto maior a densidade, maior o índice A. O mapa de densidade de lineamentos foi elaborado a partir dos lineamentos traçados por Athayde (2013) na escala 1:600.000. Os índices foram determinados a partir de intervalos quartílicos, variando de 6 (valores abaixo do primeiro quartil) até 9 (valores acima do terceiro quartil).
O parâmetro S (solo) foi caracterizado com base na classificação dos solos quanto à capacidade de infiltração, segundo critério adotado pela CPRM (2014) para elaboração do Mapa de Capacidade de Infiltração dos Solos do Brasil. A Tabela 2 apresenta os tipos de solo da região de estudo e a classificação quanto à capacidade de infiltração dos solos pela CPRM. Em posse do mapa de solos do Paraná (EMBRAPA, 2009), Figura 3, foi elaborado o mapa de capacidade de infiltração da área de estudo. Para solos com capacidade de infiltração ruim, moderada, boa e muito boa foram atribuídos os índices 3, 5, 7 e 9, respectivamente.

Tabela 2 - Tipo de solo e capacidade de infiltração

\begin{tabular}{llllll}
\hline Tipo de solo & Cap.de infitração & Índice & Tipo de solo & Cap. de infiltração & Índice \\
\hline Neossolo litólico & Moderada & 5 & Nitossolo bruno & Boa & 7 \\
Neossolo flúvico & Ruim & 3 & Argissolo vermelho & Boa & 7 \\
Neossolo regolítico & Moderada & 5 & Cambissolo háplico & Boa & 7 \\
Latossolo vermelho & Muito boa & 9 & Chernossolo rêndzico & Moderada & 5 \\
Latossolo bruno & Boa & 7 & Gleissolo háplico & Ruim & 3 \\
Nitossolo vermelho & Muito boa & 9 & Gleissolo melânico & Ruim & 3 \\
Nitossolo háplico & Boa & 7 & Organossolo háplico & Moderada & 5 \\
\hline
\end{tabular}

O parâmetro T (topografia) consiste na declividade do terreno. Para calcular a declividade, foi obtido um modelo digital de elevação do da área de estudo a partir do SRTM (Shuttle Radar Topography Mission) no formato Geotiff com resolução da célula de $90 \mathrm{~m}$. Este modelo foi adaptado para o sistema de referência brasileiro por Weber et al. (2004). Com a ferramenta Slope do ArcGIS, foi possivel obter a declividade em percentual, e os vaIores reclassificados conforme indicado pelo método DRASTIC.

Para a determinação do parâmetro I (litologia da zona vadosa), foi considerado o solo como zona vadosa do SASG. A classificação dos solos para este parâmetro foi realizada conforme proposta metodológica de Streck et al. (2008), que classifica os diferentes tipos de solo quanto a aptidão para disposição de resíduos, levando em consideração as seguintes características: profundidade, textura, pedregosidade, rochosidade, drenagem natural, erodibilidade e aptidão agrícola. A partir destas características, o solo é classificado em: adequado, regular, restrito e inadequado. A Tabela 3 apresenta a classificação dos diferentes tipos de solo da área de estudo e o índice atribuído. 
Figura 3 - Mapa de solos

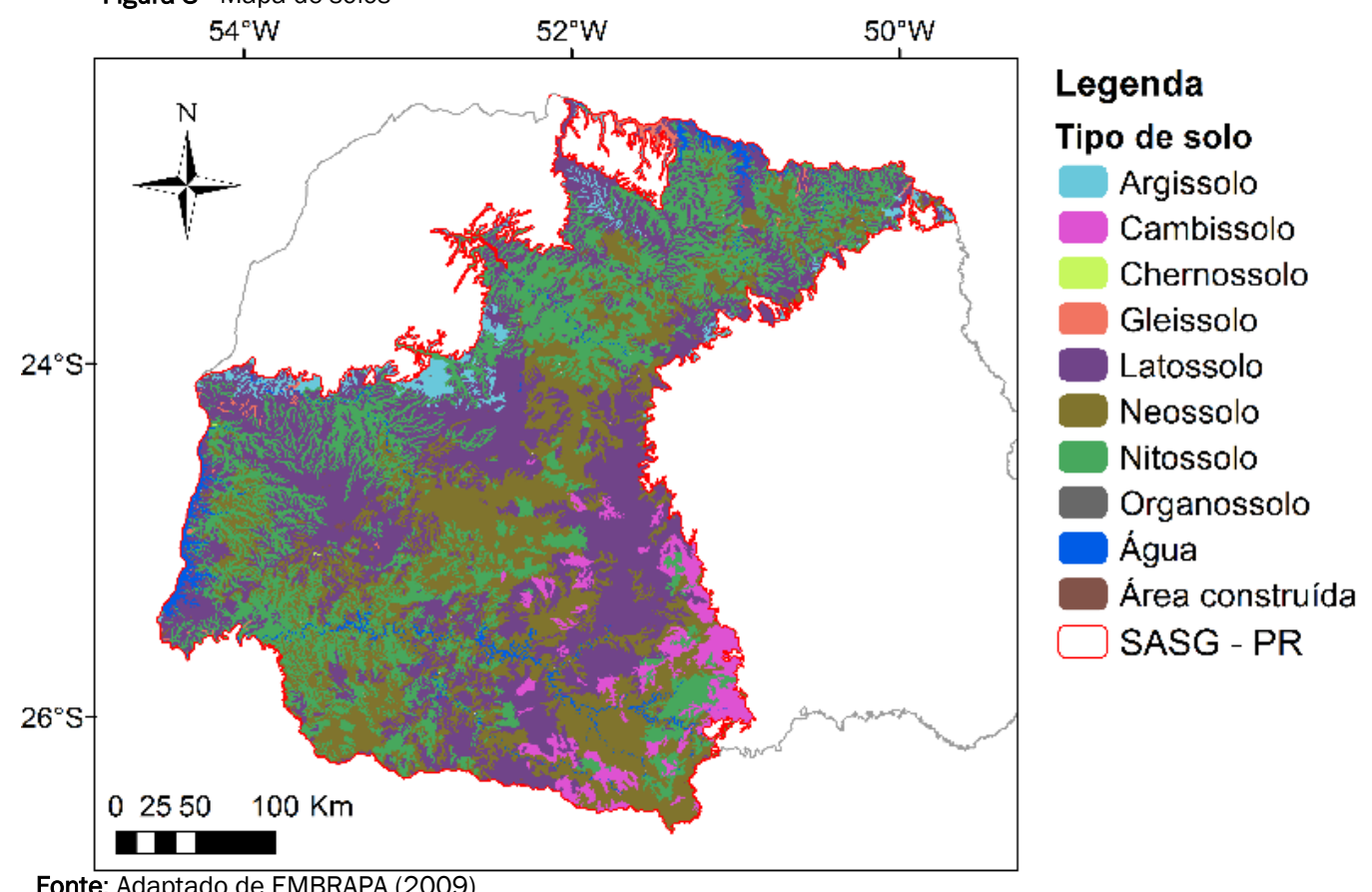

Fonte: Adaptado de EMBRAPA (2009)

Tabela 3 - Caracterização dos solos quanto à aptidão para disposição de resíduos segundo Streck et al. (2008)

\begin{tabular}{ccc}
\hline Classe & Índice & Tipos de solo \\
\hline Adequado & 2 & Latossolo argiloso; Argissolo médio/argiloso; Nitossolo. \\
Regular & 3 & Latossolo médio; Latossolo arenoso/médio. \\
Restrito & 6 & Chernossolo; Neossolo regolítico. \\
Inadequado & 8 & Cambissolo; Gleissolo; Organossolo; Neossolo flúvico e litólico.
\end{tabular}

Os aquíferos fraturados são descontínuos e anisotrópicos. Em função disto, existe uma grande dificuldade em se determinar a espessura saturada do aquífero, que é um parâmetro necessário para o cálculo da condutividade hidráulica. Portanto, o parâmetro $\mathrm{C}$ foi definido pela transmissividade do aquífero, que pode ser calculada através de ensaios de bombeamento, como uma adaptação ao método, visando a redução das incertezas. A adoção da transmissividade como parâmetro na avaliação da vulnerabilidade já foi realizada por outros autores, como Maia e Cruz (2011), cujo estudo apresentou forte correlação entre transmissividade e condutividade hidráulica.
Para o cálculo da transmissividade foram utilizados dados de 42 ensaios de bombeamento realizados na área de estudo. 0 método de cálculo utilizado foi o de Cooper e Jacob (1946). Para espacialização deste parâmetro, os resultados dos cálculos foram importados para ambiente de Sistema Geográfico de Informação e interpolados através da ferramenta Topo to Raster. A localização dos poços é apresentada na Figura 4. Os valores estão agrupados em intervalos quartílicos. 
Figura 4 - Localização dos poços para cálculo da transmissividade

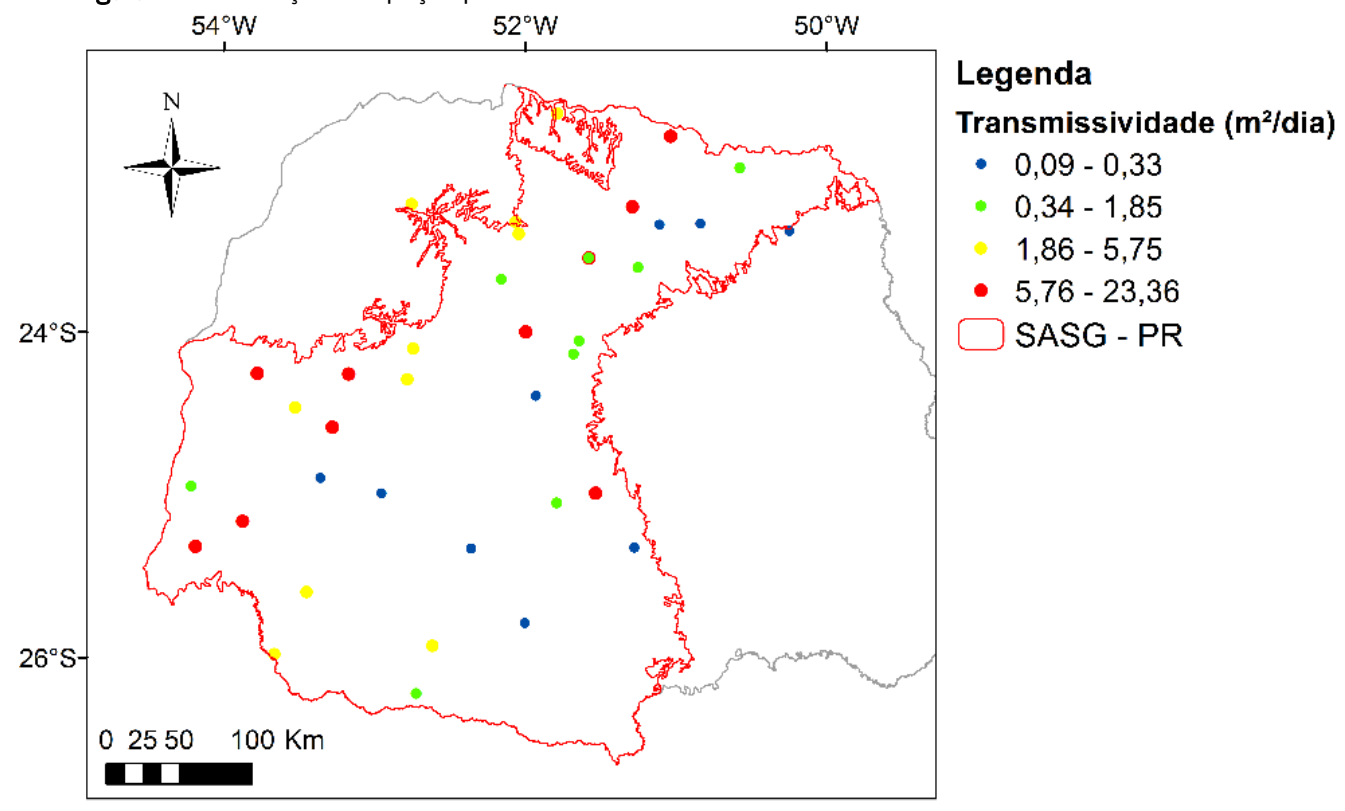

\subsection{Avaliação da vulnerabilidade pelo método GOD}

O método GOD (FOSTER e HIRATA, 1988) para avaliação da vulnerabilidade é amplamente utilizado devido a sua simplicidade de aplicação, uma vez que utiliza apenas três parâmetros: grau de confinamento da água subterrânea; ocorrência de estratos de cobertura; e distância até o aquífero freático. A interação entre estes três parâmetros gera um índice representado por valores de zero a um, indicando classes de vulnerabilidade insignificante $(0$ a 0,1$)$, baixa $(0,1$ a 0,3$)$, média $(0,3$ a 0,5$)$, alta $(0,5$ a $0,7)$ ou extrema $(0,7$ a 1,0$)$.

Para o mapeamento da vulnerabilidade pelo método GOD, os índices G, O e D foram multiplicados através da ferramenta Raster Calculator do ArcGIS. Em seguida, o valor gerado foi reclassificado para que se enquadre nas faixas de vulnerabilidade definidas pelo método.

Para a determinação do parâmetro $\mathrm{G}$ (grau de confinamento da água subterrânea), foi considerada a espessura média dos solos. Esta adaptação foi realizada, pois o SASG pode ser considerado como um aquífero não confinado coberto a livre, sendo a espessura dos solos um critério de cobertura. Os dois extremos considerados foram os solos mais espessos (nitossolo e latossolo), sendo atribuído o índice 0,6 (livre coberto) e o menos espesso (neossolo), sendo atribuído valor 1 (aquífero livre). Os demais tipos de solos considerados em classes intermediárias (argissolo, organossolo, chernossolo, cambissolo e gleissolo), de acordo com a espessura média de cada um.

Para a avaliação do índice 0 , o solo foi considerado como primeira barreira de passagem dos contaminantes, portanto, pode ser considerado como camada de cobertura. Uma vez que o contaminante ultrapassa essa barreira, infiltra pelas fraturas e atinge o aquífero. No entanto, a abordagem foi feita diferente daquela realizada ao parâmetro G, pois foram consideradas outras características além da espessura. Esta classificação foi baseada na proposta de Streck et al.(2008), conforme já descrito no item 3.1 para o método DRASTIC. Foram atribuídos os índices 0,$4 ; 0,6 ; 0,8$ e 1,0 para as classes de solo adequado, regular, restrito e inadequado, respectivamente.

Para a classificação do índice $D$, foi adotado o mesmo procedimento para o método DRASTIC com intervalos de classes conforme proposto pelo método GOD.

Para a comparação entre os métodos, foi realizada uma normalização dos mapas, de modo a facilitar a análise. Para isso, os índices de vulnerabilidade DRASTIC foram divididos por 230, para que, desta forma, variem de 0 a 1, como no método GOD. Além disso, foi gerado um histograma de frequências que relaciona índice de vulnerabilidade e número de pixels para melhor análise comparativa.

\section{RESULTADOS E DISCUSSÃO}

A Figura 5 apresenta os mapas dos parâmetros dos índices DRASTIC e o mapa final de vulnerabilidade. Os valores de cada parâmetro e o percentual de sua ocorrência na área de estudo são apresentados na Tabela 4. 0 mapa de vulnerabilidade apresentou as seguintes classes de vulnerabilidade: baixa $(5,01 \%)$, moderada (83,13\%) e elevada (11,85\%). A variabilidade espacial dos índices de vulnerabilidade se deve principalmente aos parâmetros D (profundidade do nível estático) e T (topografia), pois são os que apresentam maior quantidade de intervalos de classe. A dispersão das zonas de vulnerabilidade baixa e elevada no mapa se deve à escala regional do estudo, com elevada variabilidade espacial dos parâmetros. Além disso, a forte anisotropia típica em um aquífero fraturado pode influenciar na variabilidade espacial dos resultados, com destaque ao parâmetro D (profundidade do nível estático, que pode sofrer grandes variações em pouca distância.

O mapa de vulnerabilidade pelo método GOD, por sua vez, apresentou maior ocorrência de vulnerabilidade baixa $(66,21 \%)$, seguido de vulnerabilidade extrema (18,81\%), alta (14,32\%) e, em menor ocorrência, vulnerabilidade moderada $(0,67 \%)$. Os índices referentes a cada um dos três parâmetros bem como seu percentual de ocorrência na área de estudo são apresentados na Tabela 5. A Figura 6 apresenta os mapas dos parâmetros e 
o mapa de vulnerabilidade pelo método GOD. Os graus mais elevados de vulnerabilidade ocorrem principalmente na Bacia do Rio Iguaçu, devido a baixos valores de profundidade do nível estático associados à presença de neossolos, que são pouco espessos.

A baixa ocorrência de vulnerabilidade moderada apresentada pelo método GOD é decorrente da pouca variabilidade dos parâmetros, principalmente $\mathrm{G}$ e 0 , em que os valores intermediários ocorrem com pouca frequência. Além disso, ambos os parâmetros G e 0 são caracterizados com base no Mapa de Solos que possui escala reduzida, e, consequentemente, menor grau de detalhe.

Tabela 4 - Síntese dos índices DRASTIC e percentual de ocorrência na área de estudo

\begin{tabular}{|c|c|c|c|}
\hline Faixa & Índice & Peso & \% da área \\
\hline \multicolumn{4}{|c|}{ D - Profundidade do nível estático (m) } \\
\hline $0-1,5$ & 10 & 5 & 22,68 \\
\hline $1,5-4,6$ & 9 & 5 & 13,03 \\
\hline $4,6-9,1$ & 7 & 5 & 22,86 \\
\hline $9,1-15,2$ & 5 & 5 & 26,56 \\
\hline $15,2-22,8$ & 3 & 5 & 13,57 \\
\hline $22,8-30,5$ & 2 & 5 & 1,25 \\
\hline$>30,5$ & 1 & 5 & 0,06 \\
\hline \multicolumn{4}{|c|}{ R - Recarga (mm/ano) } \\
\hline $101,6-177,8$ & 6 & 4 & 6,18 \\
\hline $177,8-254$ & 8 & 4 & 3,64 \\
\hline$>254$ & 9 & 4 & 90,18 \\
\hline \multicolumn{4}{|c|}{ A - Meio aquífero - Densidade de lineamentos $\left(\mathrm{km} / \mathrm{km}^{2}\right)$} \\
\hline$<1^{\circ}$ quartil $(0-0,55)$ & 6 & 3 & 10,65 \\
\hline Entre o $1^{\circ}$ e $2^{\circ}$ quartil $(0,55-0,7)$ & 7 & 3 & 31,17 \\
\hline Entre o $2^{\circ}$ e $3^{\circ}$ quartil $(0,7-0,8)$ & 8 & 3 & 36,01 \\
\hline$>3^{\circ}$ quartil $(0,8-1,2)$ & 9 & 3 & 22,17 \\
\hline \multicolumn{4}{|c|}{ S - Solo - Capacidade de infiltração } \\
\hline Ruim & 3 & 2 & 3,02 \\
\hline Moderada & 5 & 2 & 27,71 \\
\hline Boa & 7 & 2 & 13,08 \\
\hline Muito boa & 9 & 2 & 56,18 \\
\hline \multicolumn{4}{|c|}{ T - Topografia - Declividade (\%) } \\
\hline$>18$ & 1 & 1 & 15,24 \\
\hline $12-18$ & 3 & 1 & 15,86 \\
\hline $6-12$ & 5 & 1 & 35,57 \\
\hline $2-6$ & 9 & 1 & 28,06 \\
\hline $0-2$ & 10 & 1 & 5,27 \\
\hline \multicolumn{4}{|c|}{ I - Zona vadosa - Aptidão dos solos para disposição de resíduos } \\
\hline Adequado & 2 & 5 & 29,51 \\
\hline Regular & 3 & 5 & 38,17 \\
\hline Restrito & 6 & 5 & 0,01 \\
\hline Inadequado & 8 & 5 & 32,32 \\
\hline \multicolumn{4}{|c|}{ C - Transmissividade $\left(\mathrm{m}^{2} /\right.$ dia $)$} \\
\hline$<1^{\circ}$ quartil $(0-0,7)$ & 3 & 3 & 25,00 \\
\hline Entre o $1^{\circ}$ e $2^{\circ}$ quartil $(0,7-2,4)$ & 5 & 3 & 25,00 \\
\hline Entre o $2^{\circ}$ e $3^{\circ}$ quartil $(2,4-5,1)$ & 7 & 3 & 25,00 \\
\hline$>3^{\circ}$ quartil $(2,4-23,4)$ & 9 & 3 & 25,00 \\
\hline
\end{tabular}


Figura 5 - Mapas dos parâmetros e de vulnerabilidade pelo método DRASTIC

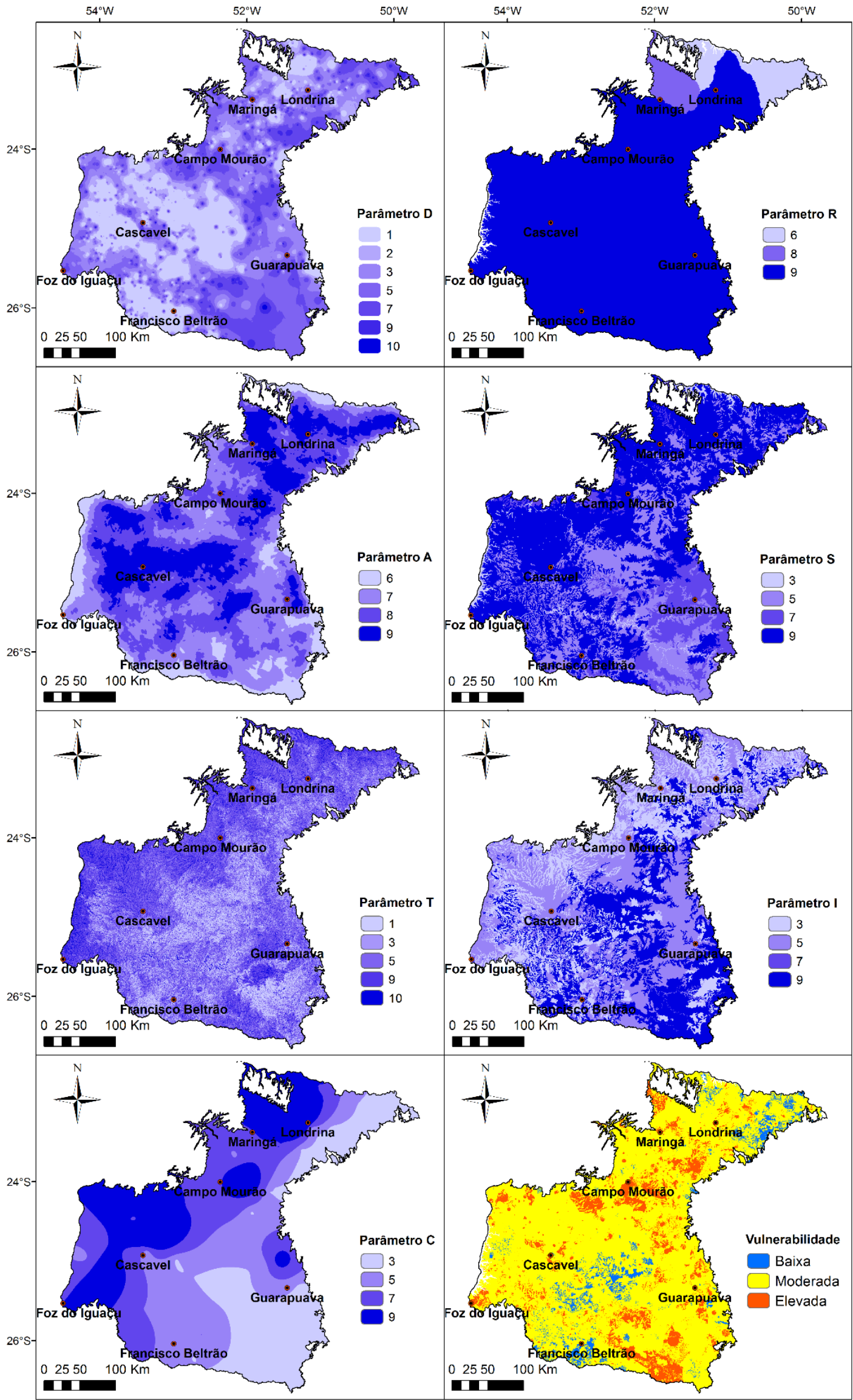


Tabela 5 - Síntese dos índices GOD e percentual de ocorrência na área de estudo

\begin{tabular}{|c|c|c|}
\hline Faixa & Índice & \% da área \\
\hline \multicolumn{3}{|c|}{ G - Grau de confinamento } \\
\hline Livre (neossolo) & 1,0 & 27,71 \\
\hline Cambissolo e gleissolo & 0,9 & 4,60 \\
\hline Organossolo e chernossolo & 0,8 & 0,02 \\
\hline Argissolo & 0,7 & 2,58 \\
\hline Livre coberto (latossolo e nitossolo) & 0,6 & 65,10 \\
\hline \multicolumn{3}{|c|}{ O - Ocorrência de estratos de cobertura } \\
\hline Adequado & 0,4 & 29,51 \\
\hline Regular & 0,6 & 38,17 \\
\hline Restrito & 0,8 & 0,01 \\
\hline Inadequado & 1,0 & 32,32 \\
\hline \multicolumn{3}{|c|}{ D - Distância até o nível d'água (m) } \\
\hline$>50$ & 0,6 & 8,17 \\
\hline $20-50$ & 0,7 & 34,60 \\
\hline $5-20$ & 0,8 & 55,38 \\
\hline$<5$ & 0,9 & 1,85 \\
\hline
\end{tabular}

Figura 6 - Mapas dos parâmetros e de vulnerabilidade pelo método GOD

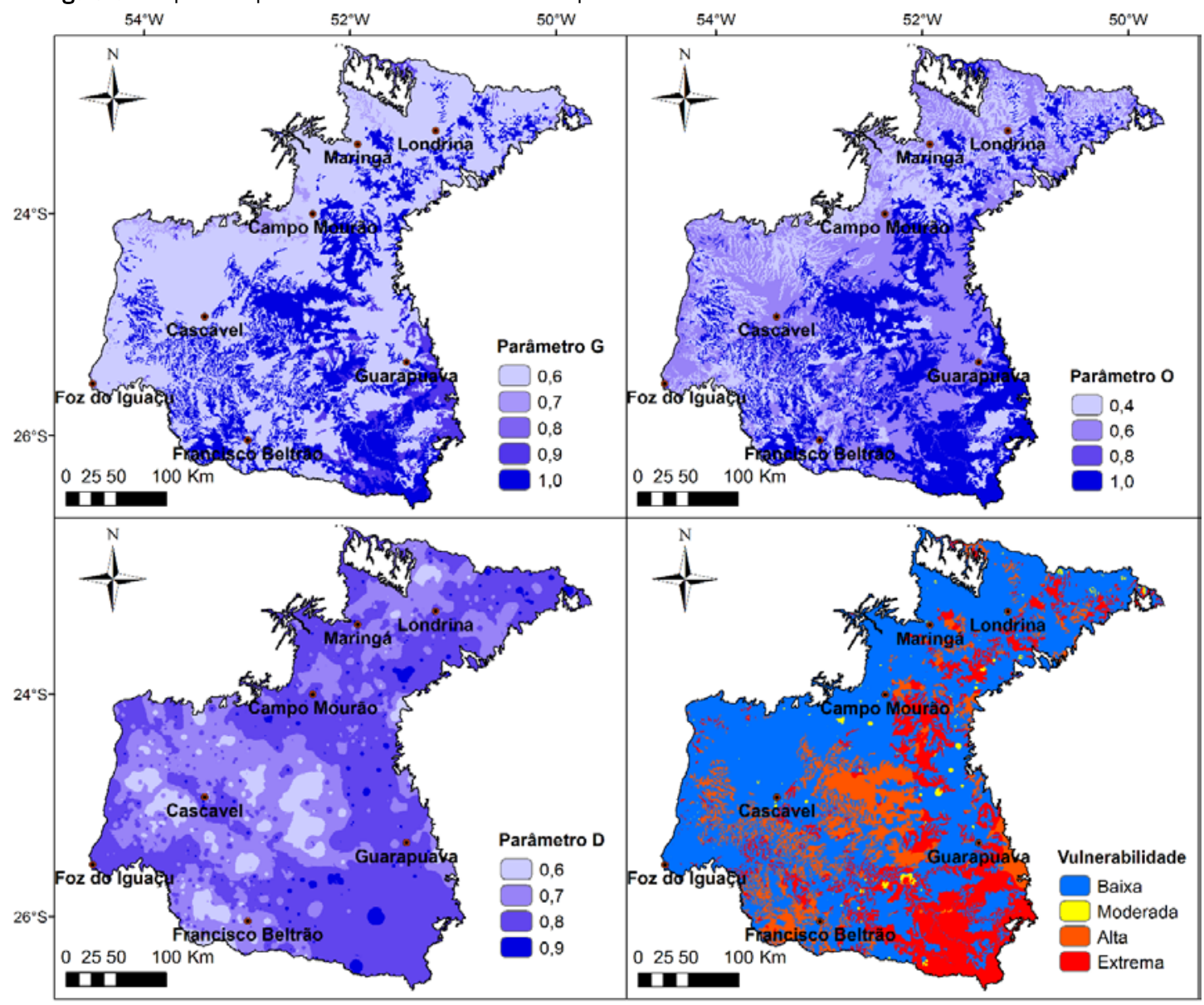

\subsection{Análise integrada dos métodos}

Os mapas com índices normalizados são apresentados na Figura 7. Estes mapas indicam uma incompatibilidade numérica entre os métodos, entretanto, algumas semelhanças na forma podem ser observadas em decorrência da utilização de parâmetros em comum. Quando calculada a diferença relativa entre os valores dos pixels pelos métodos DRASTIC e o GOD, tem-se um valor médio de 53\%. Apenas 11,4\% da área apresenta diferença relativa inferior a $10 \%$, que representa as regiões cuja vulnerabilidade é definida por parâmetros mútuos aos métodos.

O comportamento dos índices de vulnerabilidade de cada método pode ser observado nos histogramas da Figura 8. Enquanto o DRASTIC apresenta uma distribuição bastante regular, com valores intermediários mais frequentes, aproximando-se de uma 
distribuição normal, o GOD apresenta comportamento completamente diferente, com valores concentrados nos dois extremos do gráfico.

As diferenças apresentadas pelos métodos se devem ao fato de que o DRASTIC utiliza uma parametrização mais complexa em sua avaliação, em relação ao GOD, como observado anteriormente. A escassez de valores intermediários do método GOD está associada principalmente à baixa ocorrência de valores intermediários dos parâmetros $\mathrm{G}$ e $\mathrm{O}$, resultantes da baixa densidade de dados disponíveis e empregados na análise e também pela parametrização simplificada. Além disso, as diferenças podem estar associadas ao método de cálculo do índice de vulnerabilidade. Enquanto no DRASTIC é feita uma soma ponderada dos índices, no método GOD os valores são multiplicados entre si, o que resulta em uma resposta matemática distintas, tornando os valores difíceis de serem comparados.

A simplicidade do método GOD é uma vantagem, haja vista que requer menos dados e, consequentemente, menos recursos humanos e financeiros para obtenção das informações necessárias ao estudo. Entretanto, a simplificação pode causar aumento na incerteza dos resultados, pois não considera fatores importantes que favorecem o transporte de contaminantes para o aquífero como a recarga e a topografia, por exemplo. 0 método DRASTIC, por sua vez, é um modelo mais robusto e requer uma grande quantidade de informações para ser aplicado, o que pode torná-lo, muitas vezes, muito custoso. Porém, apresenta a vantagem de ser mais confiável, pois considera sete parâmetros em sua análise. Entretanto, a utilização de dados inconsistentes ou de baixa qualidade pode fragilizar a análise como um todo de ambos os métodos, sendo que, este problema possui maior probabilidade de ocorrência na utilização do método DRASTIC, uma vez que este utiliza uma grande quantidade de informações. Portanto, havendo dúvidas quanto à qualidade das informações, métodos mais simples podem ser mais adequados.

Os resultados de vulnerabilidade aqui apresentados são úteis, pois podem subsidiar primeiras ações relacionadas à gestão das águas subterrâneas no Estado e também sinalizar áreas de alerta. Entretanto, haja vista que este estudo foi realizado em escala regional, estudos em escalas de maior detalhe devem ser realizados preliminarmente a qualquer ação de controle do uso e ocupação do solo.

Figura 7 - Mapas de índices normalizados de vulnerabilidade

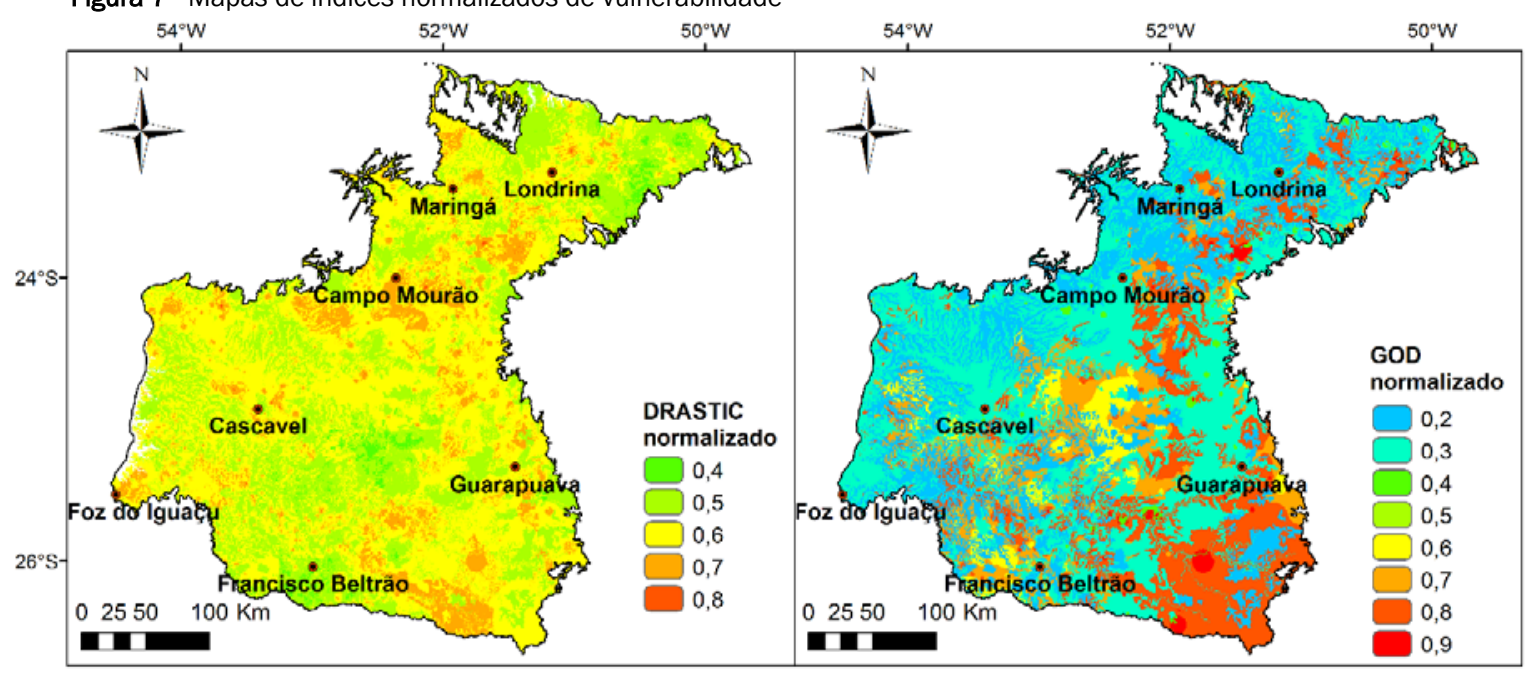

Figura 8 - Histograma de frequência dos índices DRASTIC e GOD

Histograma de frequências DRASTIC

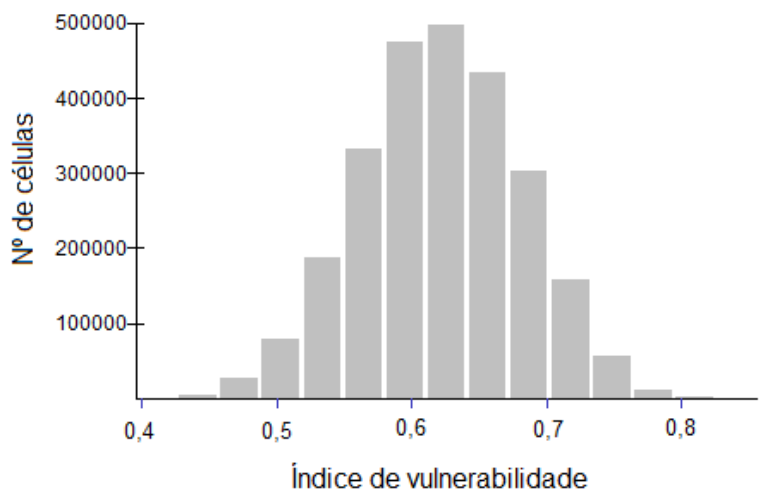

Histograma de frequências

GOD

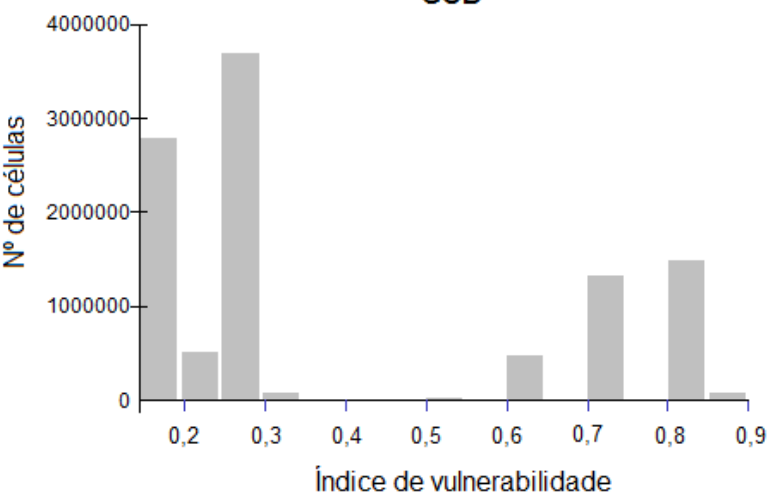




\section{CONCLUSÃO}

Neste trabalho, a avaliação da vulnerabilidade do SASG foi obtida pelos métodos DRASTIC e GOD. Como resultado desta comparação de métodos observou-se uma diferença numérica significativa, que pode estar relacionada à quantidade de parâmetros utilizados pelos métodos, e pela diferença no procedimento matemático de obtenção dos índices de vulnerabilidade. Cabe destacar que apesar das diferencias supracitada, as regiões que foram classificadas como maior ou menor vulnerabilidade coincide em ambos os métodos.

O método DRASTIC apresentou um maior grau de detalhe, com uma distribuição espacial mais suave dos valores, enquanto o GOD apresentou baixa definição espacial e variação abrupta de vulnerabilidade devido à simplificada parametrização e baixa densidade de dados. Apesar do método DRASTIC apresentar melhores resultados, o GOD possui a vantagem de depender de poucas informações para sua análise, sendo, portanto, mais exequível.

É necessário enfatizar que estes resultados devem ser utilizados com cautela, e que estudos de detalhe devem ser realizados, preliminarmente a qualquer intervenção e/ou planejamento, com intuito de caracterizar o local em escala adequada. No entanto, os resultados aqui apresentados são úteis, pois podem subsidiar as primeiras tomadas de decisões relacionadas à gestão dos recursos hídricos subterrâneos no estado do Paraná.

\section{AGRADECIMENTOS}

Os autores agradecem ao CNPq pelo apoio mediante projeto universal $n^{\circ} 446685 / 2014-3$, intitulado: Mapeamento multifinalitário no Sistema Aquífero Serra Geral, Estado do Paraná: áreas potenciais à captação para consumo humano, e vulnerabilidade natural à contaminação do aquífero, coordenado pelo Prof. Dr. Gustavo Barbosa Athayde (Instituto de Pesquisa Hidráulicas UFRGS).

\section{REFERÊNCIAS}

ALBINET, M.; MARGAT, E. J. Cartographie de la vulnerabilite a la pollution des nappes d'eau souterraine. Bulletin BRGM 2ndseries, Orleans, v. 4, n. 3, p. $13-22,1970$.

ALLER,L., et al. DRASTIC: a standardized system for evaluating groundwater pollution potential using hydrogeologic settings. U.S Environmental Protection Agengy, Oklahoma, 622 p., 1987.
ATHAYDE, G. B. Compartimentação Hidroestrutural do Sistema Aquífero Serra Geral (SASG) no Estado do Paraná, Brasil. Tese de doutorado, UFPR, Curitiba, 2013.

ATHAYDE, G. B; ATHAYDE, C. V. M. Hidrogeologia do Sistema Aquífero Serra Geral no Estado do Paraná. Revista brasileira de águas subterrâneas, v. 29, n 3, p 315 - 333, 2015

CIVITA, M. Le Carte della Vulnerabilità degli acquiferi all inquinamento: Teoria and pratica. Pitagora Editrice, Bologna, 1994.

COOPER, H. H.; JACOB, C. E. A generalized graphical method for evaluating formation constants and summarizing well field history. Am. Geophys. Union Trans., v. 27, p. 526-534, 1946.

CPRM - Mapa hidrogeológico do Brasil ao milionésimo. Pernambuco, 2014 - Escala 1:5.000.000.

ECKHARDT, K. How to Construct Recursive Digital Filters for Baseflow Separation. Hydrological Process, n. 19, p. 507-515, 2005.

EMBRAPA - Mapa de Solos do Paraná. Paraná, 2009 - Escala 1:600.000. ESRI. ArcGIS 10.3 for Desktop. Environmental Systems Research Institute, Redlands, California, 2014.

FOSTER, S. S.; HIRATA, R.Groundwater pollution risk assessment: a methodology using available data. Pan American Center for Sanitary Engineering and Environmental Sciences (CEPIS). Lima, Peru, 1988.

FRANCÉS, A. et al. Development and Application in the Alentejo Region of a Method to Assess the Vulnerability of Groundwater to Difuse Agriculture Pollution: the Susceptibility Index. In:THIRD INTERNATIONAL CONFERENCE ON FUTURE GROUNDWATER RESOURCES AT RISK,Lisboa, Geosystem Center IST, 2001

LICHT, O. A. B. O Grupo Serra Geral no Estado do Paraná. Serviço Geológico do Paraná - Mineropar, Curitiba, 2013.

MAIA, P.H.P.; CRUZ, M.J.M. Um Novo Método para Avaliar a Vulnerabilidade de Aqúiferos. Braz. J. Aquat. Sci. Techno., v. 15, n. 2, p 29 - 40, 2011.

MENESES, L. F. et al. Elaboração de Mapa de Vulnerabilidade dos Aquíferos Superiores no Município de João Pessoa-PB através de Técnicas de Geoprocessamento. Revista Tecnológica, Fortaleza, v.30, n.1, p. 123-132, 2009

SANEPAR, Companhia Paranaense de Saneamento. Notícias de 31 de março de 2015, Curitiba, 2015. Disponível em <http://site.sanepar.com.br/noticias/aquiferos-fornecem-21-da-agua-tratada-consumida-pelos-clientes>. Acesso em 6 de novembro de 2015.

STRECK, E. V. et al. Solos do Rio Grande do Sul. 2. ed. Porto Alegre: EMATER/RS - ASCAR, 2008.

TUSHAAR, N. S. et al. Groundwater: a global assessment of scale and significance. In: WATER FOR FOOD WATER FOR LIFE, Anais... p. 395-423, 2007. 